\title{
Mosaicism of activating FGFR3 mutations in human skin causes epidermal nevi
}

\author{
Christian Hafner, ${ }^{1}$ Johanna M.M. van Oers, ${ }^{2}$ Thomas Vogt, ${ }^{1}$ Michael Landthaler, ${ }^{1}$ Robert Stoehr, ${ }^{3}$ \\ Hagen Blaszyk, ${ }^{4}$ Ferdinand Hofstaedter, ${ }^{5}$ Ellen C. Zwarthoff, ${ }^{2}$ and Arndt Hartmann ${ }^{5}$

\begin{abstract}
1Department of Dermatology, University of Regensburg, Regensburg, Germany. 2Department of Pathology, Josephine Nefkens Institute, Erasmus MC, Rotterdam, The Netherlands. ${ }^{3}$ Department of Urology, University of Regensburg, Regensburg, Germany. ${ }^{4}$ Department of Pathology, University of Vermont College of Medicine, Burlington, Vermont, USA. ${ }^{5}$ Institute of Pathology, University of Regensburg, Regensburg, Germany.
\end{abstract}

\begin{abstract}
Epidermal nevi are common congenital skin lesions with an incidence of 1 in 1,000 people; however, their genetic basis remains elusive. Germline mutations of the FGF receptor 3 (FGFR3) cause autosomal dominant skeletal disorders such as achondroplasia and thanatophoric dysplasia, which can be associated with acanthosis nigricans of the skin. Acanthosis nigricans and common epidermal nevi of the nonorganoid, nonepidermolytic type share some clinical and histological features. We used a SNaPshot multiplex assay to screen 39 epidermal nevi of this type of 33 patients for 11 activating FGFR3 point mutations. In addition, exon 19 of FGFR3 was directly sequenced. We identified activating FGFR3 mutations, almost exclusively at codon 248 (R248C), in 11 of 33 (33\%) patients with nonorganoid, nonepidermolytic epidermal nevi. In 4 of these cases, samples from adjacent histologically normal skin could be analyzed, and FGFR3 mutations were found to be absent. Our results suggest that a large proportion of epidermal nevi are caused by a mosaicism of activating FGFR3 mutations in the human epidermis, secondary to a postzygotic mutation in early embryonic development. The $\mathrm{R} 248 \mathrm{C}$ mutation appears to be a hot spot for FGFR3 mutations in epidermal nevi.
\end{abstract}

\section{Introduction}

Epidermal nevi show a prevalence of about 1 in 1,000 people and can be divided into either nonorganoid (keratinocytic) types or organoid types characterized by hyperplasia of adnexal structures such as sebaceous glands, sweat glands, and hair follicles. Epidermal nevi of the common, nonorganoid and nonepidermolytic type are benign skin lesions and may vary in their extent from a single (usually linear) lesion to widespread and systematized involvement (Figure 1). They may be present at birth or develop early during childhood as localized epidermal thickening with hyperpigmentation, frequently following the lines of Blaschko. This suggests that epidermal nevi may be due to mosaicism resulting from postzygotic mutations in keratinocytes. Mutations of keratins 1 and 10 were shown to be responsible for a rare subgroup of epidermal nevi, the linear epidermolytic hyperkeratosis $(1,2)$. Another variant of epidermal nevi, the congenital hemidysplasia with ichthyosiform nevus and limb defects (CHILD) nevus, is caused by NADPH steroid dehydrogenase-like protein (NSDHL) mutations (Xq28) and represents a functional $\mathrm{X}$ chromosomal mosaicism (3). However, the genetic basis of the much more common nonorganoid, nonepidermolytic keratinocytic epidermal nevi remains elusive.

The FGF receptor (FGFR) family comprises 4 major transmembrane receptor tyrosine kinases (FGFR1-4) and is involved in embryogenesis, angiogenesis, and tissue homeostasis (4). The FGFR3 gene contains 19 exons encoding an extracellular region for ligand binding composed of 3 Ig-like domains, a hydrophobic transmembrane domain and 2 cytoplasmic tyrosine kinase

Nonstandard abbreviations used: $\mathrm{ACH}$, achondroplasia; $\mathrm{CS}$, Crouzon syndrome; FGFR3, fibroblast growth factor receptor 3; $\mathrm{HCH}$, hypochondroplasia; SADDAN, severe achondroplasia with developmental delay and acanthosis nigricans $\mathrm{TD}$, thanatophoric dysplasia.

Conflict of interest: The authors have declared that no conflict of interest exists. Citation for this article: J. Clin. Invest. 116:2201-2207 (2006). doi:10.1172/JCI28163. domains (Figure 2). Alternative splicing of the second half of the IgIII-like domain (exon 8 versus exon 9) results in the isoforms IIIb and IIIc. The isoforms show different ligand specificity and tissue expression. FGFR3 IIIb is mainly expressed in epithelial cells while FGFR3 IIIc is predominantly found in mesenchymal cells (5). This paper assigns all codon numbers to the open reading frame of the FGFR3 IIIb isoform. More than 20 FGFs are known as ligands now (4). The interaction of the ligand with the receptor requires the presence of sulfated glycosaminoglycans such as heparin and leads to the dimerization of the receptor with consecutive phosphorylation of intracellular tyrosine residues in the kinase domain and activation of intracellular signaling pathways. The autophosphorylation sites of FGFR3 represent potential binding sites for signaling proteins, for example, with phosphotyrosine binding (PTB) and Src homology 2 (SH2) domains. Activation of the cytoplasmic region causes phosphorylation of Shp2, PLC $\gamma$, ERK1/2, and STAT3 and also PI3K activation (4). FGFRs can also be activated by interaction with EphA4, another receptor tyrosine kinase, which demonstrates the complexity of FGFR signaling (6).

Activating germline mutations of the FGFR3 gene result in dwarfism, severe skeletal dysplasia, and craniosynostosis syndromes such as achondroplasia $(\mathrm{ACH})$, hypochondroplasia $(\mathrm{HCH})$, thanatophoric dysplasia (TD), Crouzon syndrome (CS), Muenke syndrome (MS), and SADDAN (severe achondroplasia with developmental delay and acanthosis nigricans) syndrome (7-10). These activating mutations result in negative chondrocytic growth regulation of the epiphyseal plates of long bones, causing dwarfism (11). Identical mutations are found in different cancer entities and probably provide proliferative signals (4). FGFR3 signaling in mutated cells is poorly understood, but previous studies provide some insights. All known missense mutations causing TD I create an unpaired cysteine residue (12-14), such as the R248C and S249C mutations. These mutations are localized in the extracellular domain at the linker region between the Ig-like domains II and III while other 

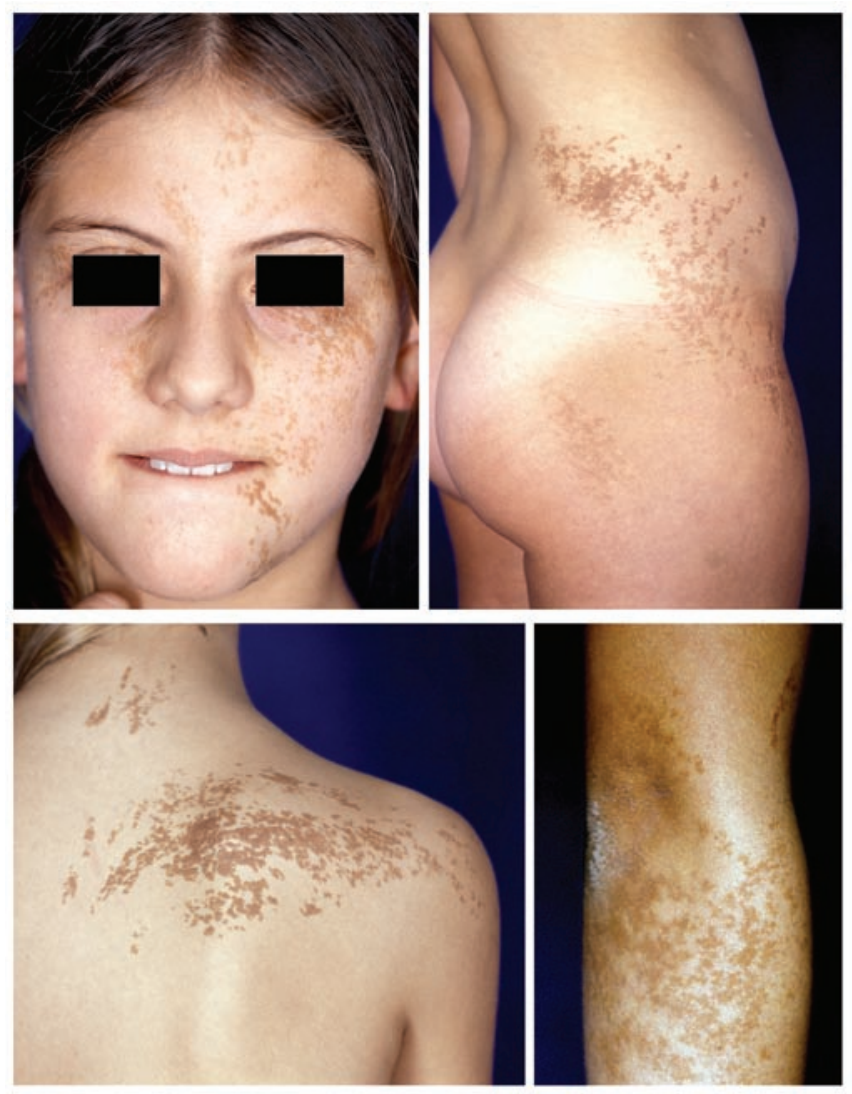

mutations causing unpaired cysteine residues such as G372C are localized at the junction of the extracellular and the transmembrane domain of FGFR3. The newly created unpaired cysteine amino acid allows the formation of disulfide bonds between the extracellular domains of 2 receptors, resulting in homodimerization, increased tyrosine phosphorylation, and ligand-independent constitutive receptor activation $(15,16)$. Similar mechanisms were also shown for other receptor tyrosine kinases, the erythropoietin receptor and the epidermal growth factor receptor $(17,18)$. Constitutive activation in cells bearing the G372C or S373C mutation results in high basal phosphorylation with significantly increased constitutive levels of MAPK phosphorylation and c-fos transcription, probably caused by mutant homodimer FGFR3 complexes (19). Other mutations, such as G382R, which is found in about $97 \%$ of $\mathrm{ACH}$ patients, affect the transmembrane domain and lead

\section{Figure 1}

Patient 29 displayed a systematized epidermal nevus of the common soft type with involvement of the face (bilateral), the right scapular region, the right arm, the right hip, and the right thigh. Abnormalities of the skeletal or nervous system were not present. A biopsy was taken from the epidermal nevus of the right forearm. This epidermal nevus revealed an R248C FGFR3 gene mutation. DNA isolated from the blood of this patient revealed WT status at codon 248 , excluding a germline mutation.

to the formation of hydrogen bonds between 2 FGFRs, resulting in constitutive receptor activation (20). The A393E mutation in $\mathrm{CS}$ also affects the transmembrane domain and results in FGFR3 dimer stabilization, measured by the change in the free energy of the dimerization, thus largely increasing the fraction of dimers (21). A third class of mutations affect the tyrosine kinase domain, such as the K652E mutation in TD II or the N542K mutation in $\mathrm{HCH}$ (11). These mutations likely cause conformational changes in the activation loop that activate the receptor tyrosine kinase activity and downstream ERK1/2 (22). Other studies suggest that FGFR3 mutations may delay the downregulation and ligand-mediated internalization of the receptor (23). The phosphorylated immature form of the mutant receptor accumulates in the endoplasmic reticulum and fails to be degraded (24). The different degree of receptor activation seems to correlate with the severity of the phenotype. Stronger activation of the receptor by ligand-independent dimerization via disulfide bonds in TD patients determines the more severe phenotype compared with other skeletal dysplasia syndromes such as ACH and $\mathrm{HCH}(15,25)$.

Somatic activating FGFR3 mutations have been identified in $40 \%$ of human seborrheic keratoses (26) and in several human cancers (4), including multiple myeloma (27), urothelial carcinoma (28), cervix carcinoma (29), and colorectal carcinoma (30). Some of the skeletal dysplasia syndromes (TD, CS, SADDAN) caused by FGFR3 mutations are also characterized by marked thickening of the epidermis. This skin lesion, termed acanthosis nigricans, and epidermal nevi share similar histological features, including acanthosis and papillomatosis $(31,32)$. Herein we investigate the role of FGFR3 mutations in common nonorganoid, nonepidermolytic keratinocytic epidermal nevi.

\section{Results}

We analyzed 39 common nonepidermolytic, nonorganoid keratinocytic epidermal nevi of 33 patients using a SNaPshot multiplex assay that covered 11 FGFR3 point mutations described in skeletal dysplasia syndromes and cancer entities (Figure 2). The following subtypes of keratinocytic epidermal nevi were

FGFR3 IIIb

\section{Figure 2}

FGFR3 gene. The position of the mutations covered by the SNaPshot multiplex assay is indicated. Codons are numbered according to the FGFR3 IIIb isoform; potential mutations of the stop codon 809 in exon 19 associated with TD I were analyzed by direct sequencing. C, C-terminus; Ig I, Ig II, Ig III, Ig-like domains I-III; N, $\mathrm{N}$-terminus; TM, transmembrane domain; TKI, TKII, tyrosine kinase domains I-II.

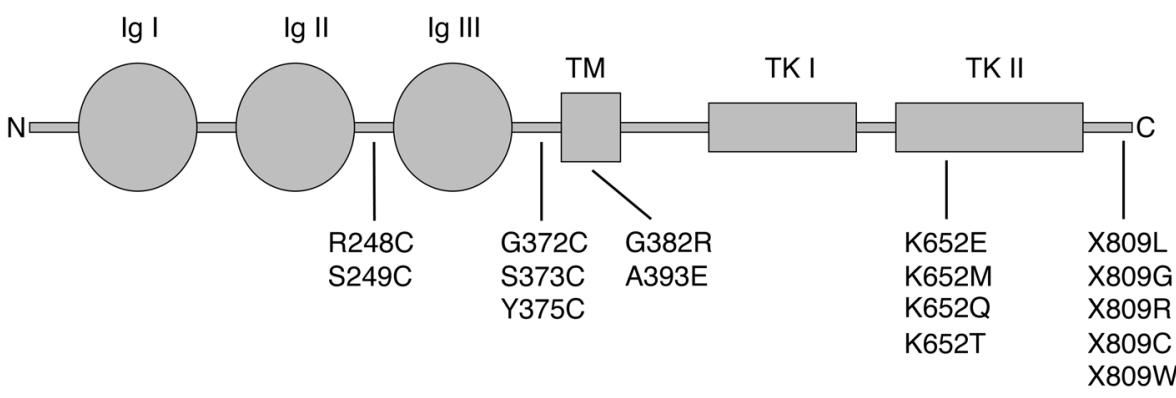


Table 1

FGFR3 mutations in epidermal nevi

\begin{tabular}{|c|c|c|c|c|c|c|c|}
\hline No. & Sex & Age (yr) & Site & Subtype & Nevus & Control & Syst. \\
\hline 1 & $\mathrm{~F}$ & 1 & Head & Seborrheic & WT & - & No \\
\hline 2 & $M$ & 59 & Head & Acro-verruc & WT & - & - \\
\hline 3 & $\mathrm{~F}$ & 9 & Left axilla & Common hard & $\mathrm{R} 248 \mathrm{C}$ & WT & - \\
\hline 4 & $M$ & 13 & Neck & Seborrheic & WT & - & No \\
\hline 5 & $\mathrm{~F}$ & 36 & Right axilla & Common soft & $\mathrm{R} 248 \mathrm{C}$ & - & No \\
\hline 6 & $M$ & 17 & Head & Common soft & WT & - & - \\
\hline 7 & $M$ & 7 & Head & Common soft & WT & - & - \\
\hline 8 & M & 21 & Head & Common soft & WT & - & - \\
\hline 9 & M & 15 & Head & Common soft & WT & - & - \\
\hline 10 & $\mathrm{~F}$ & 15 & Right shoulder & Common hard & WT & - & - \\
\hline $11 a$ & $M$ & 22 & Head & Common soft & WT & - & No \\
\hline $11 b$ & $M$ & 22 & Head & Common soft & WT & - & No \\
\hline 12 & $M$ & 20 & Left groin & Common soft & $\mathrm{R} 248 \mathrm{C}$ & - & No \\
\hline 13 & $\mathrm{~F}$ & 13 & Back & Common soft & WT & - & - \\
\hline 14 & $\mathrm{~F}$ & 23 & Back & Common soft & WT & - & - \\
\hline 15 & $\mathrm{~F}$ & 5 & Left axilla & Common soft & G372C/G382R & - & - \\
\hline 16 & $\mathrm{~F}$ & 2 & Neck & Common soft & WT & - & No \\
\hline 17 & M & 6 & Neck & Common soft & $\mathrm{R} 248 \mathrm{C}$ & WT & Yes \\
\hline 18 & M & 16 & Head & Common soft & WT & - & No \\
\hline 19 & $\mathrm{~F}$ & 27 & Neck & Common soft & WT & - & - \\
\hline 20 & $\mathrm{~F}$ & 25 & Right shoulder & Common soft & WT & - & - \\
\hline 21 & M & 29 & Head & Common hard & $\mathrm{R} 248 \mathrm{C}$ & WT & No \\
\hline 22 & $\mathrm{~F}$ & 20 & Right arm & Common soft & $\mathrm{R} 248 \mathrm{C}$ & - & - \\
\hline 23 & $\mathrm{~F}$ & 16 & Trunk & Common hard & WT & - & No \\
\hline 24 & $M$ & 31 & Head & Common soft & WT & - & No \\
\hline 25 & $\mathrm{~F}$ & 18 & Back & Seborrheic & WT & - & - \\
\hline 26 & M & 9 & Neck & Common soft & $\mathrm{R} 248 \mathrm{C}$ & - & Yes \\
\hline 27 & $M$ & 14 & Head & Seborrheic & WT & - & No \\
\hline 28 & $\mathrm{~F}$ & 9 & Head & Acro-verruc & WT & - & No \\
\hline 29 & $\mathrm{~F}$ & 11 & Right forearm & Common soft & $\mathrm{R} 248 \mathrm{C}$ & - & Yes \\
\hline 30 & $\mathrm{~F}$ & 15 & Head & Common hard & WT & - & No \\
\hline 31 & $\mathrm{~F}$ & 16 & Neck & Common soft & WT & - & - \\
\hline 32 & $M$ & 23 & Back & Common soft & $\mathrm{R} 248 \mathrm{C}$ & WT & No \\
\hline $33 a$ & $\mathrm{~F}$ & 13 & Neck & Common soft & $\mathrm{R} 248 \mathrm{C}$ & - & Yes \\
\hline $33 b$ & $\mathrm{~F}$ & 13 & Neck & Common soft & $\mathrm{R} 248 \mathrm{C}$ & - & Yes \\
\hline $33 c$ & $\mathrm{~F}$ & 13 & Neck & Common soft & $\mathrm{R} 248 \mathrm{C}$ & - & Yes \\
\hline $33 d$ & $\mathrm{~F}$ & 13 & Neck & Common soft & $\mathrm{R} 248 \mathrm{C}$ & - & Yes \\
\hline $33 e$ & $\mathrm{~F}$ & 13 & Neck & Common soft & $\mathrm{R} 248 \mathrm{C}$ & - & Yes \\
\hline $33 f$ & $\mathrm{~F}$ & 13 & Neck & Common soft & R248C & - & Yes \\
\hline
\end{tabular}

Age, age at time of biopsy; site, site of biopsy; control, DNA from clinically and histologically normal epidermis adjacent to the epidermal nevus; syst., systematized epidermal nevus; M, male; F, female; acro-verruc, acrokeratosis verruciformis-like; R248C, exon 7, codon 248, CGC to TGC with Arg to Cys; G372C, exon 10, codon 372, GGC to TGC with Gly to Cys; G382R, exon 10, codon 382, GGG to AGG with Gly to Arg. Codon numbers refer to the open reading frame of the FGFR3 IIlb isoform.

included in the study: 28 common soft type, 5 common hard type, 4 seborrheic-like type, and 2 acrokeratosis verruciformislike type. We identified activating FGFR3 mutations in 11 of 33 (33\%) patients and in 16 of 39 (41\%) epidermal nevi (Table 1). In 15 of 16 epidermal nevi (94\%), the mutation occurred in exon 7 at codon 248 (CGC to TGC), resulting in an Arg to Cys amino acid substitution. In one epidermal nevus (patient 15), a double mutation was found in exon 10 at codon 372 (GGC to TGC), resulting in a Gly to Cys amino acid substitution, and at codon 382 (GGG to AGG), resulting in a Gly to Arg amino acid substitution. The epidermal nevus with the double mutation was a common soft-type nevus and showed no histological abnormalities compared with the other epidermal nevi. All activating
FGFR3 mutations were found in commontype epidermal nevi (14 common soft type, and 2 common hard type).

In 4 patients (patients $3,17,21$, and 32) with an FGFR3 mutation (R248C) in the epidermal nevus, clinically and histologically normal epidermis adjacent to the nevus showed a WT codon 248 , suggesting an epidermal mosaicism of the FGFR3 mutation and a strong genotype-phenotype correlation (Figure 3). In 1 patient (patient 29 ) with a systematized epidermal nevus displaying the R248C mutation (biopsy was taken from the right forearm), additional genomic DNA was isolated from blood. The DNA revealed the WT codon 248 , thus excluding a germline mutation. Multiple intraindividual epidermal nevi biopsies could be analyzed in 2 patients. One patient (patient 11a, b) did not show any FGFR3 mutations. The other patient (patient 33a-e) underwent ablative laser treatment of the common soft-type epidermal nevus at the right side of the neck (Figure 4). Before treatment, 6 of the scattered brownish papules were curetted for FGFR3 mutation analysis. All 6 samples, which were spatially distant from each other, revealed the R248C mutation in the SNaPshot analysis.

All FGFR3 mutations detected so far in skin lesions (seborrheic keratoses and epidermal nevi) are associated with TD, CS, and SADDAN syndrome in the germline. The SNaPshot multiplex assay covered all mutations responsible for these syndromes except for the stop codon mutation X809L/G/R/C/W in exon 19 causing TD I (Figure 2). For 20 epidermal nevi (nevi of patients 1 , $2,5,6,7,8,9,10,11[\mathrm{a}, \mathrm{b}], 14,16,17,20,23,27$, $28,30,31$, and 32), DNA was available for further analysis. We additionally sequenced exon 19 of these samples. Seventeen of the 20 epidermal nevi had not shown any FGFR3 mutations in the $\mathrm{SNaPshot}$ analysis. However, no further FGFR3 mutations were detected in exon 19.

Since epidermal nevi represent a heterogeneous skin disorder, we also studied an organoid type of epidermal nevus, the sebaceous nevus. This nevus is found almost exclusively on the scalp or face and is usually present at birth. Sebaceous nevi are histopathologically characterized by the presence of large numbers of mature sebaceous glands and papillomatous hyperplasia of the epidermis. We analyzed 13 sebaceous nevi for FGFR3 point mutations using the SNaPshot multiplex assay (Table 2). In contrast to the common nonorganoid keratinocytic nevi, this organoid type of epidermal nevus revealed no FGFR3 mutations. Thus, activating FGFR3 mutations, especially the frequently detected R248C mutation, appear to be specific for common nonorganoid keratinocytic nevi.

\section{Discussion}

Our results indicate that a significant number of epidermal nevi of the common, nonorganoid, and nonepidermolytic keratinocytic type are caused by postzygotic mutations in the FGFR3 gene, which 


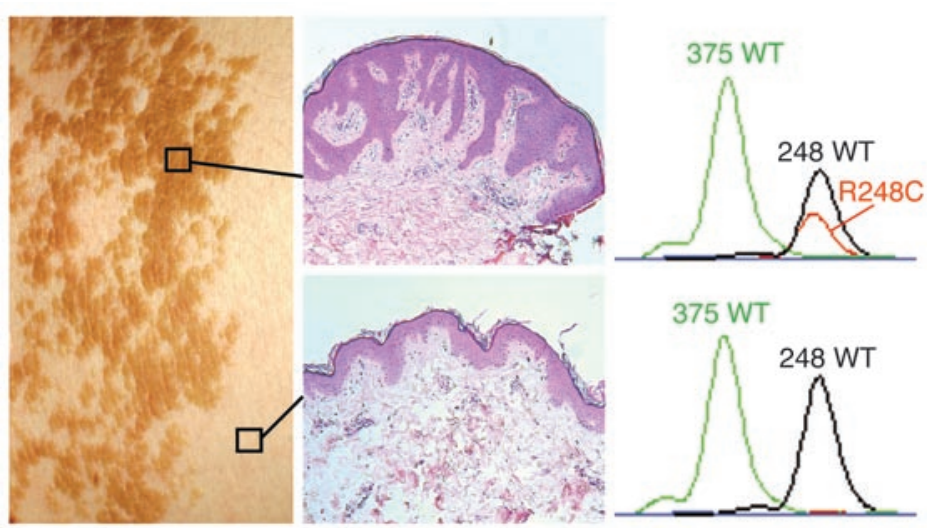

Figure 3

Patient 32 had a common soft-type epidermal nevus on his back. Two biopsies were taken from the epidermal nevus and the adjacent normal skin. The epidermal nevus histologically showed the typical acanthosis and papillomatosis (H\&E staining; original magnification, $\times 40$ ) and an R248C mutation in the SNaPshot analysis. In contrast, the clinically and histologically normal epidermis revealed a WT status for codon 248. This result suggests a strong genotype-phenotype correlation and the presence of a mosaicism of the FGFR3 mutation in the epidermis of this patient.

likely occur early in embryonic development in a single keratinocytic stem cell. The descendants of the mutated stem cell will migrate along the lines of Blaschko, resulting in the linear or whirled cutaneous patterns usually observed in epidermal nevi (33).

Several findings support the notion that the detected FGFR3 mutations are causative for the development of epidermal nevi: (a) The detected mutations R248C and, in one case, G372C are known to occur in TD I patients and act as dominant germline mutations in this lethal skeletal dysplasia syndrome. Affected individuals are often stillborn or die as neonates. It has previously been postulated that such lethal mutations may only survive by mosaicism (34). The $\mathrm{R} 248 \mathrm{C}$ mutation results in strong constitutive and ligand-independent receptor activation (15), excluding the possibility that the detected mutations have no functional consequence in the investigated skin samples. (b) Mutations responsible for TD I (including R248C and G372C) have been shown to cause another benign skin lesion, the seborrheic keratosis (26). Epidermal nevi and seborrheic keratoses share many clini$\mathrm{cal}$ and histopathological features. Another activating mutation (S249C) associated with TD I causes thickening of the skin and verrucous skin tumors in a mouse model. The murine skin lesions resemble seborrheic keratoses (and likewise epidermal nevi) clinically and histologically (26). (c) A TD I patient with an R248C mutation and long-term survival developed acanthosis nigricans, a skin disorder that is clinically and histologically very similar to epidermal nevi (10). Both acanthosis nigricans and epidermal nevi

\section{Figure 4}

Patient 33 revealed a common soft-type epidermal nevus at the right side of the neck consisting of scattered brownish papules following the lines of Blaschko. Before ablative laser treatment, 6 papules distant from each other were curetted for FGFR3 mutation analysis. The green peaks represent WT codon 375, the black peaks WT codon 248. The R248C mutation is characterized by a red peak. All 6 samples displayed the R248C mutation. In the bottom right corner, control DNA with WT codon 248 is shown. show acanthosis, papillomatosis, hyperkeratosis, and basal hyperpigmentation $(31,32)$. A potential overlap between the 2 skin disorders is acknowledged since both acanthosis nigricans type of epidermal nevus and nevoid acanthosis nigricans have been described $(32,35,36)$. In our series of epidermal nevi, an acanthosis nigricans-like type was not included. A female patient with a mosaicism of R248C (25\% of the blood lymphocytes were affected by the mutation) developed disseminated thickening and hyperpigmentation of the skin consistent with acanthosis nigricans (37). (d) Some patients with epidermal nevus syndrome showed typical keratinocytic epidermal nevi, skeletal abnormalities, and the occurrence of urothelial carcinoma at an early age (38-40). This correlation of epidermal nevus and urothelial carcinoma is thought to be nonstochastic. FGFR3 mutations are frequent events in papillary urothelial carcinoma $(29,41)$. These findings and the skeletal abnormalities reminiscent of skeletal dysplasia syndromes strongly suggest that such patients feature a mosaicism of activating FGFR3 mutations, which in turn cause epidermal nevi of the skin, skeletal changes, and bladder cancer. (e) In one patient of our series (patient 33), multiple intraindividual samples of the epidermal nevus localized on the neck could be analyzed. All 6 samples revealed the same R248C mutation, suggesting the presence of a scattered FGFR 3 mutation mosaicism in the skin of this patient following the lines of Blaschko. (f) The strongest support that the detected mutations are indeed causative for epidermal nevi is that 4 patients with an R248C mutation in the epidermal nevus showed the WT allele in clinically and histologically normal epidermis adjacent to the epidermal nevus. This also suggests mosaicism of an FGFR3 mutation in the epidermis and a strong genotype-phenotype correlation.

In our series, 15 of 16 epidermal nevi with an FGFR3 mutation displayed the $\mathrm{R} 248 \mathrm{C}$ mutation, resulting in a cytosine-thymine substitution (CGC to TGC). This $\mathrm{C}$ to $\mathrm{T}$ transition is a typical example of deamination of methylated cytosines leading to $\mathrm{CpG}$ dinucleotide depletion. The reason for this mutational hot spot is unknown. One could speculate that the development of epi-

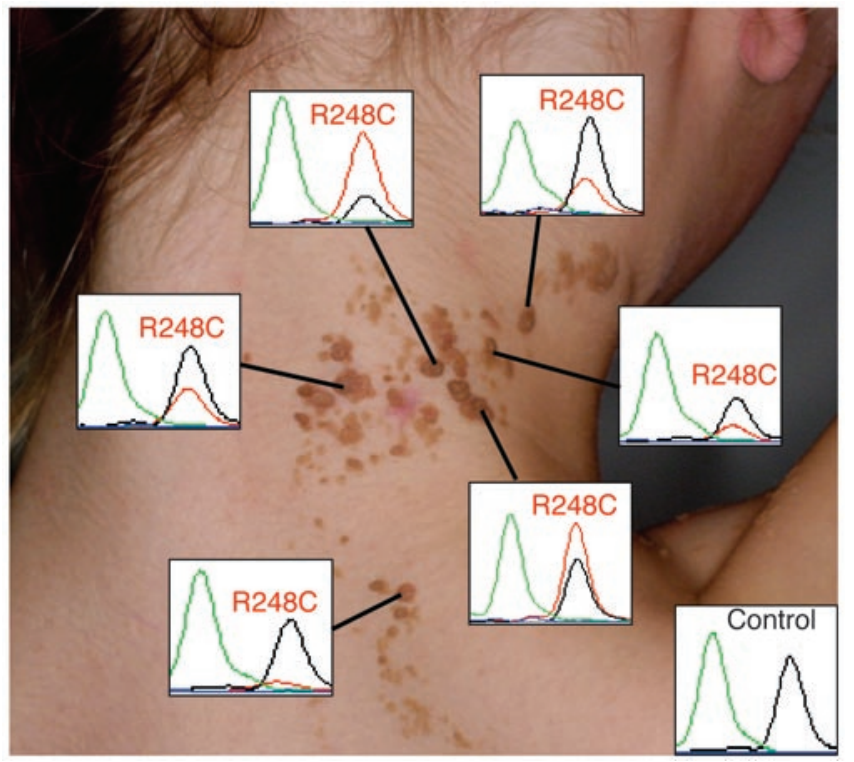


Table 2

FGFR3 mutation analysis in sebaceous nevi

\begin{tabular}{lcccc} 
No. & Sex & Age (yr) & Site & Sebaceous nevus \\
1 & $\mathrm{M}$ & 17 & Head & WT \\
2 & $\mathrm{M}$ & 15 & Head & WT \\
3 & $\mathrm{~F}$ & 7 & Head & WT \\
4 & $\mathrm{M}$ & 12 & Head & WT \\
5 & $\mathrm{M}$ & 33 & Head & WT \\
6 & $\mathrm{M}$ & 17 & Head & WT \\
7 & $\mathrm{M}$ & 7 & Head & WT \\
8 & $\mathrm{M}$ & 14 & Head & WT \\
9 & $\mathrm{M}$ & 13 & Head & WT \\
10 & $\mathrm{~F}$ & 16 & Head & WT \\
11 & $\mathrm{M}$ & 12 & Head & WT \\
12 & $\mathrm{M}$ & 32 & Head & WT \\
13 & $\mathrm{M}$ & 13 & Head & WT \\
\hline
\end{tabular}

dermal nevi with acanthosis and papillomatosis of the epidermis requires strong activation of the receptor and not all activating FGFR3 mutations are capable of this strong activation. Indeed, Naski et al. found that the R248C mutation activates the receptor more strongly than the G382R mutation responsible for $\mathrm{ACH}(15)$. Stronger activation of the receptor is thought to result in a more severe phenotype in TD compared with other skeletal dysplasia syndromes (14). According to this theory, mosaicism of FGFR3 mutations other than R248C may also be present in the skin, but they may be insufficient to induce epidermal nevi. This hypothesis is supported by Logie et al. (26), who found that seborrheic keratoses sharing some histological characteristics with epidermal nevi are caused by acquired somatic FGFR 3 mutations. All somatic mutations detected so far in seborrheic keratoses (R248C, S249C, G372C, S373C, Y375C, K652E, and K652M) are associated with TD and SADDAN syndrome in germline. Remarkably, none of the mutations associated with $\mathrm{ACH}$ or $\mathrm{HCH}$ were found in this series of 62 seborrheic keratoses. Patients with TD and SADDAN syndrome show acanthosis nigricans whereas $\mathrm{ACH}$ and $\mathrm{HCH}$ are usually not associated with this skin lesion except for in 1 reported case (42). ACH is the most common cause of dwarfism in humans, with an incidence of $1: 15,000$ to $1: 40,000$ of live births, and the mutated nucleotide 1138 in ACH is thought be the most mutable nucleotide in the human genome described so far $(9,43)$. The R248C mutation is the most common mutation in TD (44), but it remains elusive why other mutations causing TD and SADDAN syndrome (S249C, S373C, Y375C, K652E, K652M) were not detected in our series of epidermal nevi.

Epidermal nevus syndromes are characterized by epidermal nevi, abnormalities of the skeletal and nervous system, and rarely, some associated cancer entities $(45,46)$. The occurrence of papillary bladder cancer and skeletal abnormalities in epidermal nevus syndromes (38-40) suggests that FGFR3 is a promising candidate gene for epidermal nevus syndromes that may be caused by a more widespread mosaicism of FGFR3 mutations. If the FGFR3 mosaicism involves the germ cells in those patients, the offspring should show a TD phenotype. However, we did not find any reports of patients with systematized epidermal nevi or epidermal nevus syndrome and an offspring with TD in the literature. Another interesting fact is that acanthosis nigricans is predominantly observed in intertriginous areas in skeletal dysplasia syndromes. The reason for this preferential localization remains unclear since the entire skin carries the mutation. There seems to be a preponderance of intertriginous localization (neck, axilla, groin) also in the epidermal nevi with FGFR3 mutations in our series (Table 1). Additional cofactors in intertriginous areas may favor the development of acanthosis and papillomatosis on the basis of activating FGFR3 mutations.

One epidermal nevus in our series displayed a double mutation, the G372C mutation known from TD I and the typical G382R mutation known from $\mathrm{ACH}$. It remains elusive whether the G372C mutation alone, which causes a stronger activation of the FGFR3 receptor according to the associated skeletal dysplasia syndromes (13-15), was able to cause the epidermal nevus or whether the combination of both mutations was necessary for the induction of the nevus. The possibility that the nevus was mainly caused by the G382R mutation seems unlikely since ACH patients, in contrast to TD patients, usually do not develop acanthosis nigricans $(10,42)$. Double mutations in the FGFR3 gene have been reported in urothelial carcinoma $(28,47,48)$. To our knowledge, germline compound heterozygosity for mutations associated with TD and $\mathrm{ACH}$ has not been described. The epidermal nevus carrying the double mutation displayed no histopathological abnormalities.

Each of the major 3 cell types present in our microdissected samples (keratinocytes, dendritic cells, and melanocytes) may theoretically be the carrier of the detected mutations, but keratinocytes represent the likely host cell type. Logie et al. studied a mouse model in which the S249C mutation (causing TD I) was targeted to the basal layer of the epidermis using the keratin 5 promoter (26). Keratin 5 is a marker for basal keratinocytes and not expressed in melanocytes or dendritic cells. The transgenic mice developed thickening of the skin and verrucous skin tumors with histological features similar to epidermal nevi. The basal hyperpigmentation mediated by melanocytes would be a secondary phenomenon if the mutations affect keratinocytes. However, further studies are needed to prove this hypothesis.

It is unclear why the same mutation (R248C) can cause both seborrheic keratoses and epidermal nevi. FGFR3 mutations exhibit pleiotropic effects ranging from inherited skeletal dysplasia syndromes and benign skin tumors to cancer. The cell type-specific involvement of different signaling pathways such as Ras/MAPK and STAT as well as the FGFR3-dependent recruitment of cell-specific second receptors such as EphA4 (6) may determine the effect of activating FGFR3 mutations in each cell type (4).

Mutations of FGFR3 in the urothelium are significantly associated with benign urothelial papillomas (49) and with low-grade and low-stage pTa G1/2 tumors, which rarely progress (41, 48, 50). These findings support the concept that mutations of the FGFR3 IIIb isoform can induce proliferation and tumor formation but are associated with a low malignant potential. This would be consistent with our findings of FGFR3 mutations in epidermal nevi that represent a benign skin disorder and show signs of hyperproliferation (namely acanthosis and papillomatosis) but bear no malignant potential.

Several small molecule tyrosine kinase inhibitors of FGFR3, such as PKC412, PD173074, and SU5402, are already available. They have been used in vitro and in animal models to inhibit the growth of multiple myeloma cell lines with activating FGFR3 mutations (51-54). PKC412 is currently being evaluated in phase II trials for acute myeloid leukemia patients (55). This molecule effectively inhibits the tyrosine kinase activity of FGFR3, as shown by the 
inhibition of proliferation in hematopoietic cells transformed by a mutant FGFR3 gene (55). A broader use of this drug in other disorders associated with an increased activity of FGFR3 has been suggested. This may also include benign skin lesions such as seborrheic keratoses (26) and epidermal nevi. The current standard therapy for epidermal nevi and seborrheic keratoses is surgery or ablative laser treatment, which is often associated with irreversible scar formation. Topical application of tyrosine kinase inhibitors of FGFR3 may obviate the need for surgical intervention.

In summary, a large proportion of human epidermal nevi are caused by mosaicism of postzygotic activating FGFR3 mutations in the human epidermis. The R248C mutation appears to be a hot spot for FGFR3 mutations in epidermal nevi. Additional studies are needed to investigate other regions of FGFR3 and different receptor tyrosine kinases as possible mutational targets in epidermal nevi and elucidate functional aspects of enhanced FGFR3 signaling in the skin.

\section{Methods}

Microdissection. Thirty-nine histologically confirmed nonepidermolytic epidermal nevi of the common nonorganoid type of 33 patients with varying degrees of skin involvement were retrieved from the histology files of the Department of Dermatology, University of Regensburg. Informed consent for the scientific use of the material and photographs had been obtained from all patients according to the guidelines of the ethics committee of the University of Regensburg and the Declaration of Helsinki. Classical symmetrical acanthosis nigricans in addition to the epidermal nevi was not seen in any of the study patients, and no signs of skeletal dysplasia or associated cancer were observed. We classified the different subtypes of epidermal nevi according to a previous study (32). The common keratinocytic type was subdivided into soft and hard types according to the degree of hyperkeratosis. The characteristics of the patients and their nevi are shown in Table 1. We also investigated an organoid epidermal nevus type, the sebaceous nevus, because epidermal nevi represent a heterogeneous group of lesions. Thirteen sebaceous nevi were retrieved from the histology files of the Department of Dermatology of the University of Regensburg (Table 2).

Sections of $10-\mu \mathrm{m}$ thickness were microdissected manually from paraffin-embedded epidermal nevi tissues with a needle under an inverted microscope. We dissected the acanthotic epidermis of the nevi containing mainly keratinocytes but also small numbers of melanocytes and dendritic cells. In sebaceous nevi, both the hypertrophic sebaceous glands and the acanthotic epidermis were dissected. Clinically and histologically normal epidermis adjacent to the nevus was microdissected in 4 patients with common keratinocytic nevi to serve as a source of control DNA.

DNA isolation. DNA was isolated following standard protocols. In brief, about 25-50 mg formalin-fixed paraffin-embedded tissue was microdissected for each sample. The microdissected tissue was digested with proteinase $\mathrm{K}$ overnight in lysis buffer, and DNA isolation was performed with the High Pure PCR Template Preparation Kit (Roche Diagnostics) accord- ing to the manufacturer's protocol. The DNA of each tissue was eluted in a volume of $200 \mu \mathrm{l}$ elution buffer. The amount of isolated DNA ranged from $5 \mu \mathrm{g}$ to $30 \mu \mathrm{g}$ for each sample.

$\mathrm{SNaPshot}$ assay. A previously described $\mathrm{SNaPshot}$ multiplex assay, based on the SNaPshot Multiplex System assay (Applied Biosystems), was used to screen for activating FGFR3 point mutations (56). We used $2 \mu \mathrm{l}$ template DNA for the multiplex PCR. The SNaPshot multiplex PCR assay can detect mutations with an input DNA amount of only $1 \mathrm{ng}$ genomic DNA (56). Three regions of interest in exons 7, 10, and 15 comprising 11 FGFR3 mutations were amplified in 1 multiplex PCR, followed by extension of mutation-specific primers with a labeled dideoxynucleotide. Two new antisense primers were added to the original assay to screen for mutations S373C (5'-T19GAGGATGCCTGCATACACAC-3') and G382R (5'-T56GAACAGGAAGAAGCCCACCC- $\left.3^{\prime}\right)$. Concentrations for those primers used in the multiplex assay were 1.0 and $0.6 \mathrm{pmol} / \mu \mathrm{l}$, respectively. Thus, screening could be performed for 11 known mutations found in bladder tumors and other noncutaneous epithelial malignancies (R248C, S249C, G372C, S373C, Y375C, G382R, A393E, K652E, K652M, K652Q, and K652T; codons are numbered according to the open reading frame of the FGFR3 IIIb isoform, which is predominantly present in epithelial cells). This mutation spectrum also covers the most frequently found FGFR3 mutations in skeletal dysplasia syndromes. Extended primers were separated by capillary electrophoresis in an automatic sequencer, and the presence or absence of a mutation was indicated by the incorporated WT or mutant labeled dideoxynucleotide. When a mutation is present, a second peak from the mutated nucleotide will appear next to the WT peak in the electropherogram (see Figures 3 and 4). However, the assay is not quantitative due to the different emission efficiencies of the labels. Mutations were confirmed by a second independent reaction.

FGFR3 sequence analysis. In addition to the SNaPshot analysis, exon 19 of the FGFR3 gene was directly sequenced. This exon contains the potential mutation at stop codon 809 associated with TD I. We were able to analyze 20 epidermal nevi. The other samples failed due to limited DNA amounts. We used the forward primer $5^{\prime}$-CCTGTCGGCGCCTTTGGAGCAG- $3^{\prime}$ and the reverse primer 5'-CAGACCAAAGCTCTGTAGCT-3' to generate a 235 bp PCR product of exon 19 containing the stop codon 809 . Sequence analysis was performed following standard protocols.

\section{Acknowledgments}

We thank our patients for their participation and Alois Eckl for the helpful support. The excellent technical work of Monika Kerscher, Kirstin van der Keur, Anne Pietryga-Krieger, Lydia Kuenzel, and Nadine Wandtke is gratefully acknowledged.

Received for publication February 8, 2006, and accepted in revised form May 16, 2006.

Address correspondence to: Christian Hafner, Department of Dermatology, University of Regensburg, Franz-Josef-Strauss-Allee 11, 93042 Regensburg, Germany. Phone: 49-941-944-9610; Fax: 49941-944-9611; E-mail: christian.hafner@klinik.uni-regensburg.de.
1. Rothnagel, J.A., et al. 1992. Mutations in the rod domains of keratins 1 and 10 in epidermolytic hyperkeratosis. Science. 257:1128-1130.

2. Paller, A.S., et al. 1994. Genetic and clinical mosaicism in a type of epidermal nevus. N. Engl. J. Med. 331:1408-1415.

3. Konig, A., Happle, R., Bornholdt, D., Engel, H., and Grzeschik, K.H. 2000. Mutations in the NSDHL gene, encoding a 3beta-hydroxysteroid dehydrogenase, cause CHILD syndrome. Am. J. Med. Genet. 90:339-346.
4. L'Hote, C.G., and Knowles, M.A. 2005. Cell responses to FGFR3 signalling: growth, differentiation and apoptosis. Exp. Cell Res. 304:417-431.

5. Scotet, E., and Houssaint, E. 1995. The choice between alternative IIIb and IIIc exons of the FGFR-3 gene is not strictly tissue-specific. Biochim. Biophys. Acta. 1264:238-242.

6. Yokote, H., et al. 2005. Trans-activation of EphA4 and FGF receptors mediated by direct interactions between their cytoplasmic domains. Proc. Natl. Acad. Sci.U. S. A. 102:18866-18871.
7. Meyers, G.A., Orlow, S.J., Munro, I.R., Przylepa, K.A., and Jabs, E.W. 1995. Fibroblast growth factor receptor 3 (FGFR3) transmembrane mutation in Crouzon syndrome with acanthosis nigricans. Nat. Genet. 11:462-464.

8. Muenke, M., et al. 1997. A unique point mutation in the fibroblast growth factor receptor 3 gene (FGFR3) defines a new craniosynostosis syndrome. Am. J. Hum. Genet. 60:555-564.

9. Vajo, Z., Francomano, C.A., and Wilkin, D.J. 2000. The molecular and genetic basis of fibroblast 
growth factor receptor 3 disorders: the achondroplasia family of skeletal dysplasias, Muenke craniosynostosis, and Crouzon syndrome with acanthosis nigricans. Endocr. Rev. 21:23-39.

10. Baker, K.M., Olson, D.S., Harding, C.O., and Pauli, R.M. 1997. Long-term survival in typical thanatophoric dysplasia type 1. Am. J. Med. Genet. 70:427-436.

11. Webster, M.K., and Donoghue, D.J. 1997. FGFR activation in skeletal disorders: too much of a good thing. Trends Genet. 13:178-182.

12. Tavormina, P.L., et al. 1995. Another mutation that results in the substitution of an unpaired cysteine residue in the extracellular domain of FGFR3 in thanatophoric dysplasia type I. Hum. Mol. Genet. 4:2175-2177.

13. Tavormina, P.L., et al. 1995. Thanatophoric dysplasia (types I and II) caused by distinct mutations in fibroblast growth factor receptor 3. Nat. Genet. 9:321-328.

14. Rousseau, F., et al. 1996. Missense FGFR3 mutations create cysteine residues in thanatophoric dwarfism type I (TD1). Hum. Mol. Genet. 5:509-512.

15. Naski, M.C., Wang, Q., Xu, J., and Ornitz, D.M. 1996. Graded activation of fibroblast growth factor receptor 3 by mutations causing achondroplasia and thanatophoric dysplasia. Nat. Genet. 13:233-237.

16. d'Avis, P.Y., et al. 1998. Constitutive activation of fibroblast growth factor receptor 3 by mutations responsible for the lethal skeletal dysplasia thanatophoric dysplasia type I. Cell Growth Differ. 9:71-78.

17. Watowich, S.S., et al. 1992. Homodimerization and constitutive activation of the erythropoietin receptor. Proc. Natl. Acad. Sci. U. S. A. 89:2140-2144.

18. Sorokin, A., Lemmon, M.A., Ullrich, A., and Schlessinger, J. 1994. Stabilization of an active dimeric form of the epidermal growth factor receptor by introduction of an inter-receptor disulfide bond. J. Biol. Chem. 269:9752-9759.

19. Adar, R., Monsonego-Ornan, E., David, P., and Yayon, A. 2002. Differential activation of cysteinesubstitution mutants of fibroblast growth factor receptor 3 is determined by cysteine localization. J. Bone Miner. Res. 17:860-868.

20. Webster, M.K., and Donoghue, D.J. 1996. Constitutive activation of fibroblast growth factor receptor 3 by the transmembrane domain point mutation found in achondroplasia. $E M B O J .15: 520-527$.

21. Li, E., You, M., and Hristova, K. 2006. FGFR3 dimer stabilization due to a single amino acid pathogenic mutation. J. Mol. Biol. 356:600-612.

22. Lievens, P.M., Roncador, A., and Liboi, E. 2006. K644E/M FGFR3 mutants activate Erk1/2 from the endoplasmic reticulum through FRS2alpha and PLCgamma-independent pathways. J. Mol. Biol. 357:783-792.

23. Monsonego-Ornan, E., Adar, R., Feferman, T., Segev, O., and Yayon, A. 2000. The transmembrane mutation G380R in fibroblast growth factor receptor 3 uncouples ligand-mediated receptor activation from down-regulation. Mol. Cell. Biol. 20:516-522.
24. Lievens, P.M., Mutinelli, C., Baynes, D., and Liboi, E. 2004. The kinase activity of fibroblast growth factor receptor 3 with activation loop mutations affects receptor trafficking and signaling. J. Biol. Chem. 279:43254-43260.

25. Bellus, G.A., et al. 2000. Distinct missense mutations of the FGFR3 lys 650 codon modulate receptor kinase activation and the severity of the skeletal dysplasia phenotype. Am. J. Hum. Genet. 67:1411-1421.

26. Logie, A., et al. 2005. Activating mutations of the tyrosine kinase receptor FGFR3 are associated with benign skin tumors in mice and humans. Hum. Mol. Genet. 14:1153-1160.

27. Chesi, M., et al. 1997. Frequent translocation $\mathrm{t}(4 ; 14)(\mathrm{p} 16.3 ; \mathrm{q} 32.3)$ in multiple myeloma is associated with increased expression and activating mutations of fibroblast growth factor receptor 3 . Nat. Genet. 16:260-264.

28. van Rhijn, B.W., et al. 2002. Novel fibroblast growth factor receptor 3 (FGFR3) mutations in bladder cancer previously identified in non-lethal skeletal disorders. Eur. J. Hum. Genet. 10:819-824.

29. Cappellen, D., et al. 1999. Frequent activating mutations of FGFR3 in human bladder and cervix carcinomas. Nat. Genet. 23:18-20.

30. Jang, J.H., Shin, K.H., and Park, J.G. 2001. Mutations in fibroblast growth factor receptor 2 and fibroblast growth factor receptor 3 genes associated with human gastric and colorectal cancers. Cancer Res. 61:3541-3543.

31. Schwartz, R.A. 1994. Acanthosis nigricans. J. Am. Acad. Dermatol. 31:1-19; quiz 20-22.

32. Su, W.P. 1982. Histopathologic varieties of epidermal nevus. A study of 160 cases. Am. J. Dermatopathol. 4:161-170.

33. Happle, R. 1993. Mosaicism in human skin. Understanding the patterns and mechanisms. Arch. Dermatol. 129:1460-1470.

34. Happle, R. 1987. Lethal genes surviving by mosaicism: a possible explanation for sporadic birth defects involving the skin. J. Am. Acad. Dermatol. 16:899-906.

35. Curth, H.O. 1976. Unilateral epidermal naevus resembling acanthosis nigricans. Br. J. Dermatol. 95:433-436.

36. Kim, M.Y., Lee, J.S., Kim, H.O., and Park, Y.M. 2004. A case of nevoid acanthosis nigricans. Acta Derm. Venereol. 84:234-235.

37. Hyland, V.J., et al. 2003. Somatic and germline mosaicism for a R248C missense mutation in FGFR3, resulting in a skeletal dysplasia distinct from thanatophoric dysplasia. Am. J. Med. Genet. A. 120:157-168.

38. Rongioletti, F., and Rebora, A. 1991. Epidermal nevus with transitional cell carcinomas of the urinary tract. J. Am. Acad. Dermatol. 25:856-858.

39. Rosenthal, D., and Fretzin, D.F. 1986. Epidermal nevus syndrome: report of association with transitional cell carcinoma of the bladder. Pediatr. Dermatol. 3:455-458.

40. Garcia de Jalon, A., et al. 2004. Epidermal naevus syndrome (Solomon's syndrome) associated with bladder cancer in a 20 -year-old female. Scand. J. Urol. Nephrol. 38:85-87.

41. Kimura, T., et al. 2001. The incidence of thanatophoric dysplasia mutations in FGFR3 gene is higher in low-grade or superficial bladder carcinomas. Cancer. 92:2555-2561.

42. Van Esch, H., and Fryns, J.E. 2004. Acanthosis nigricans in a boy with achondroplasia due to the classical Gly380Arg mutation in FGFR3. Genet. Couns. 15:375-377.

43. Wilkie, A.O. 1997. Craniosynostosis: genes and mechanisms. Hum. Mol. Genet. 6:1647-1656.

44. Passos-Bueno, M.R., et al. 1999. Clinical spectrum of fibroblast growth factor receptor mutations. Hum. Mutat. 14:115-125.

45. Vujevich, J.J., and Mancini, A.J. 2004. The epidermal nevus syndromes: multisystem disorders. J. Am. Acad. Dermatol. 50:957-961.

46. Vidaurri-de la Cruz, H., Tamayo-Sanchez, L., Duran-McKinster, C., de la Luz Orozco-Covarrubias, M., and Ruiz-Maldonado, R. 2004. Epidermal nevus syndromes: clinical findings in 35 patients. Pediatr. Dermatol. 21:432-439.

47. Jebar, A.H., et al. 2005. FGFR3 and Ras gene mutations are mutually exclusive genetic events in urothelial cell carcinoma. Oncogene. 24:5218-5225.

48. van Rhijn, B.W., et al. 2003. Molecular grading of urothelial cell carcinoma with fibroblast growth factor receptor 3 and MIB-1 is superior to pathologic grade for the prediction of clinical outcome. J. Clin. Oncol. 21:1912-1921.

49. van Rhijn, B.W., Montironi, R., Zwarthoff, E.C., Jobsis, A.C., and van der Kwast, T.H. 2002. Frequent FGFR3 mutations in urothelial papilloma. J. Pathol. 198:245-251.

50. Billerey, C., et al. 2001. Frequent FGFR3 mutations in papillary non-invasive bladder (pTa) tumors. Am. J. Pathol. 158:1955-1959.

51. Mohammadi, M., et al. 1997. Structures of the tyrosine kinase domain of fibroblast growth factor receptor in complex with inhibitors. Science. 276:955-960.

52. Trudel, S., et al. 2004. Inhibition of fibroblast growth factor receptor 3 induces differentiation and apoptosis in $\mathrm{t}(4 ; 14)$ myeloma. Blood. 103:3521-3528.

53. Paterson, J.L., et al. 2004. Preclinical studies of fibroblast growth factor receptor 3 as a therapeutic target in multiple myeloma. Br. J. Haematol. 124:595-603.

54. Grand, E.K., Chase, A.J., Heath, C., Rahemtulla, A., and Cross, N.C. 2004. Targeting FGFR3 in multiple myeloma: inhibition of $\mathrm{t}(4 ; 14)$-positive cells by SU5402 and PD173074. Leukemia. 18:962-966.

55. Chen, J., et al. 2005. FGFR3 as a therapeutic target of the small molecule inhibitor PKC412 in hematopoietic malignancies. Oncogene. 24:8259-8267.

56. van Oers, J.M., et al. 2005. A simple and fast method for the simultaneous detection of nine fibroblast growth factor receptor 3 mutations in bladder cancer and voided urine. Clin. Cancer Res. 11:7743-7748. 